\title{
Solving the copper paradox
}

There is a discrepancy between the concentration of copper in the bulk continental crust and its building blocks, basaltic arc magmas. This so-called copper paradox indicates that our understanding of continental crust formation is incomplete. The presence of a hidden copper reservoir, formed by crystallization of sulfide-rich cumulates (which form through the gravitational settling of crystalline phases during magma cooling and crystallization) at the base of thick continental arcs
(45-80 km depth), has been proposed to explain this paradox but is yet to be empirically shown.

Kang Chen from China University of Geoscience (Wuhan) and Rice University, USA, and colleagues used isotope dilution to measure the concentration of copper in a series of garnet pyroxenite xenoliths (crustal fragments brought to the surface during subsequent eruptions) from Arizona, USA. These xenoliths represent deep, sulfide-bearing cumulates, which critically contain

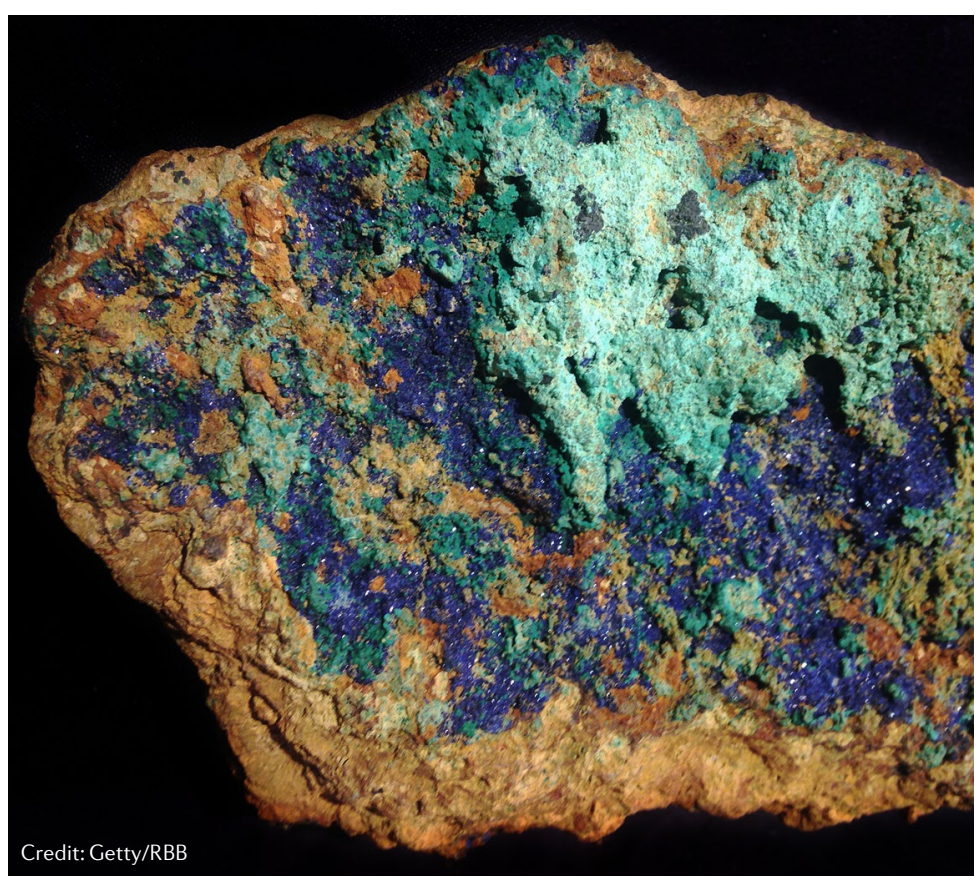

exceptionally high concentrations of copper (up to 1,000 ppm), approximately two orders of magnitude higher than observed in the bulk continental crust. Simple models of magmatic differentiation further demonstrate that the formation of crystalline sulfide minerals will strip copper from the residual melt phase and sequester it into the resulting cumulates. Thus, sulfidebearing cumulates at the base of continental arcs represent the hidden copper reservoir.

Identifying this copper-rich reservoir at the base of thick continental arcs represents an important contribution to our understanding of the copper budget of the continental crust. In addition, these observations, alongside the copper-poor nature of the bulk continental crust, indicate that sequestration of copper into these deep sulfide-bearing cumulates may represent an important component of crustal growth. Nevertheless, these results are based on analyses of copper and sulfide-rich cumulates from one location in Arizona. Additional research is needed to recognise their ubiquity beneath thick continental arcs globally.

Matthew Gleeson

ORIGINAL ARTICLE Chen, K. et al. Sulfide-bearing cumulates in deep continental arcs: The missing copper reservoir. EPSL https://doi.org/10.1016/ j.epsl.2019.115971 (2019) 